\title{
Effects of Escitalopram Administration on Face Processing in Intermittent Explosive Disorder: An fMRI Study
}

\author{
Henk Cremers*,', Royce Lee', Sarah Keedy', K Luan Phan² and Emil Coccaro' \\ 'Biological Science Division, Department of Psychiatry, University of Chicago, Chicago, IL, USA; ${ }^{2}$ Department of Psychiatry, University of Illinois at \\ Chicago, Chicago, IL, USA
}

\begin{abstract}
The neurobiological underpinnings of intermittent explosive disorder (IED) are traditionally linked to deficiencies in the serotonergic system. In this study, we investigated the effects of escitalopram, a selective serotonin reuptake inhibitor (SSRI), on brain activation during face processing. We expected that escitalopram would reduce amygdala activity in IED and in addition, we explored the effect in other social-emotional-related brain regions. A total of 17 subjects with current IED and 14 healthy controls participated in a randomized, double-blind, placebo-controlled, counterbalanced fMRI face processing study. The analysis focused on the faces compared to a fixation baseline contrast, and a factorial model with Group as between-subject and Drug as within-subject factor was tested. Group $\times$ Drug interaction effects were found in the amygdala (small volume corrected) and the left temporal parietal junction (TPJ; whole-brain corrected). Escitalopram increased amygdala activation in controls, but surprisingly not in IED. However, the TPJ showed increased activity in IED on escitalopram compared with placebo. The TPJ is associated with social-cognitive processes, such as perspective taking and empathy. The TPJ findings suggest that SSRI administration may reduce aggressive tendencies towards other people by enhancing these social-cognitive processes. Future research should further elucidate the long-term effects of SSRIs on various social-emotional tasks in IED.

Neuropsychopharmacology (2016) 4I, 590-597; doi:I0.I038/npp.2015.I87; published online 5 August 2015
\end{abstract}

\section{INTRODUCTION}

Intermittent explosive disorder (IED) is characterized by the expression of recurrent, problematic impulsive aggressive behavior (Coccaro, 2000). The degree of aggressiveness expressed during the episodes is grossly out of proportion to any precipitating psychosocial stress. IED affects 5-7\% of the US population, and is often but not necessarily comorbid with mood, anxiety, substance use, and personality disorders (Coccaro, 2012).

The neurobiological underpinnings of aggression are traditionally linked to deficiencies in the serotonergic system (5-HT; Coccaro, 1989, 2000, 2012; Lesch and Merschdorf, 2000). For instance, a negative correlation between an indicator for 5-HT transporter binding and aggression was found in personality disorder patients with IED (Coccaro et al, 2010; Coccaro, 2012) and IED patients show reduced 5-HT transporter availability in the anterior cingulate cortex (Frankle et al, 2005). Moreover, males with IED tend to increase intensity ratings of angry faces after tryptophan depletion that reduces 5-HT availability (Lee et al, 2012). Conversely, fluoxetine (a selective serotonin reuptake

* Correspondence: Dr H Cremers, Department of Psychiatry, University of Chicago, MC 3077, 584I South Maryland Avenue, L-602, Chicago, IL 60637, USA, Tel: + I 773834 4645,

E-mail: hcremers@yoda.bsd.uchicago.edu

Received 16 March 20I5; revised I5 May 20I5; accepted 3 June 20I5; accepted article preview online 24 June 2015 inhibitor (SSRI) that increases 5-HT availability) was shown to reduce impulsive aggressive behavior in patients with personality disorders and comorbid IED (Coccaro et al, 2009). Two studies in other aggressive populations (Silva et al, 2010; George et al, 2011) replicated this finding. However, despite the evidence for the central role of 5-HT, it is unknown how SSRI administration affects the neural mechanism of IED.

In an fMRI study on face processing, Coccaro et al (2007) found a stronger amygdala response to angry faces in IED compared with controls, and a correlation between amygdala activation and a life history of aggression. Moreover, IED patients showed less amygdala-orbitofrontal cortex connectivity than controls, suggesting reduced regulatory control of the prefrontal cortex. Previous work already showed that IED patients make disadvantageous choices on the Iowa gambling task, hinting at the possibility of disrupted prefrontal functioning (Best et al, 2002). The limited available research on the neural systems underlying IED so far thus points at a disrupted amygdala-prefrontal circuitry, yet a complex disorder like IED undoubtedly involves an even broader network of brain regions. A study on multicomponent cognitive behavioral therapy, consisting of coping skill training and cognitive restructuring, showed effectiveness in reducing aggressive symptoms (McCloskey et al, 2008). Such finding hints at the possible underlying involvement of social-cognitive (eg, empathy) and regulatory processes and related brain networks in IED. 
In this study, we investigate the effects of escitalopram (an SSRI) on the neural mechanisms in IED when viewing faces. Research has mostly shown that, in controls, a single-dose or short-term administration of citalopram reduces amygdala activity (Del-Ben et al, 2005; Harmer et al, 2006; Anderson et al, 2007; Murphy et al, 2009; Windischberger et al, 2010), although increases have also been found (Bigos et al, 2008). We hypothesize that escitalopram will lower amygdala activity in IED. In addition, we explore the effects of escitalopram on other regions involved in social-emotional processing.

\section{MATERIALS AND METHODS}

\section{Participants}

A total of 31 subjects completed this study and were included in the analysis. All subjects were right-handed and had normal or corrected-to-normal vision. Groups were matched on age and gender (Table 1). In all, 17 subjects met DSM-5 criteria for current IED and 14 subjects met inclusion/ exclusion criteria as a healthy control. Healthy controls had no current or past history of any psychiatric or personality disorder and none had a first-degree relative with documented history of any psychiatric disorder. Six other subjects were excluded from the analysis: three completed the first session only, one had a brain cyst, and two subjects had poor-quality fMRI data (excessive head motion or spikes, determined by visual inspection and with artifact detection software: www.nitrc.org/projects/artifact_detect). Subjects were recruited through advertisements in the community. All subjects gave written informed consent. The study was approved by our institutional review board.

\section{Diagnostic Assessment}

Diagnoses were made using information from: (1) the Structured Clinical Interview for DSM Diagnoses (SCID-IV; First et al, 1997) for syndromal disorders and the Structured Interview for the Diagnosis of DSM Personality Disorder (SIDP; Pfohl et al, 1997); (2) clinical interview by a research psychiatrist; and (3) review of all other available clinical data. Final diagnoses were assigned by team best-estimate consensus procedures involving research psychiatrists and clinical psychologists as previously described (Coccaro et al, 2014). Subjects with a current history of a substance use disorder or of a life history of bipolar disorder, schizophrenia (or other psychotic disorder), or mental retardation were excluded from study. Medical health of all subjects was documented by medical history and examination, and urine screen for illicit drugs. Supplementary Table S1 lists the current and life-time comorbidity of all IED participants.

\section{Assessment of Aggression, Impulsivity, and Psychopathic Personality}

Aggression was assessed with the Aggression score from the Life History of Aggression (LHA; Coccaro et al, 1997) that assesses history of actual aggressive behavior. Impulsivity was assessed using the Life History of Impulsive Behavior (LHIB; Coccaro and Schmidt-Kaplan, 2012) that assesses history of
Table I Participant Characteristics

\begin{tabular}{lcccc}
\hline & $\begin{array}{c}\text { Controls } \\
\text { mean (SD) }\end{array}$ & $\begin{array}{c}\text { IED } \\
\text { mean (SD) }\end{array}$ & & \\
\hline Characteristic & & & $T$ & $P$ \\
Age & $36.07(9.1)$ & $32.53(9.5)$ & 1.0 & 0.3 \\
Gender & $8 \mathrm{M} / 6 \mathrm{~F}$ & $10 \mathrm{M} / 7 \mathrm{~F}$ & & \\
LHA-self & $5.1(3.0)$ & $21.9(6.6)$ & 8.8 & $<0.00$ I \\
LHA-clinical & $3.8(2.9)$ & $23.8(7.7)$ & 9.1 & $<0.001$ \\
LHIB & $16.28(9.34)$ & $51.05(18.68)$ & 6.3 & $<0.001$ \\
PCL_total & $0.78(0.69)$ & $9.1(5.9)$ & 5.19 & $<0.001$ \\
BIS & $51.7(8.3)$ & $69.5(14.8)$ & 3.90 & $<0.001$ \\
\hline
\end{tabular}

Abbreviations: BIS, Barratt Impulsivity Scale; LHA, Lifetime History of Aggression; LHIB, Lifetime History of Impulsive Behavior; PCL-SV, Psychopathy ChecklistScreening Version.

actual impulsive behavior, and the Barratt Impulsiveness Scale (BIS-11; Barratt, 1965) that assesses impulsive tendencies as a personality trait. The Psychopathy Checklist Screening Version (PCL-SV; Hart et al, 1995) was used to assess for presence of Psychopathic Personality (PP) using a threshold of PCL-SV score $\geq 13$.

\section{Study Design}

A randomized, double-blind, placebo-controlled, counterbalanced design was used. Either placebo or escitalopram (0.375 mg/kg; typically $\sim 30 \mathrm{mg}$ per subject) was administered orally 2 to $3 \mathrm{~h}$ before the MR scans. This timing was right after the time of peak neuroendocrine response to escitalopram and right before the time of peak plasma concentration of escitalopram. Participants verbally rated their mood state on a scale ranging from $0(=$ not at all $)$ to 9 ( = extremely) for happy, angry, sad, fear, calm, and irritable. There was an interval of $\sim 1$ to 2 weeks between scan sessions ( $M=12.1$ days, SEM $=1.9$, range: 5-57 days).

The paradigm involved the explicit emotional valence recognition of facial expressions in a block-related fMRI design. Stimuli consisted of black and white photographs of human facial expressions from both the Penn Emotion Recognition task faces (Gur et al, 2002) and the Ekman faces (Ekman and Friesen, 1976). The task consisted of two functional runs, where each run included 14 blocks of $20 \mathrm{~s}$ duration. Within each run, blocks of different emotional expression were presented twice: angry (A), fearful (F), and neutral $(\mathrm{N})$. One block consisted of pictures of a radio (as a visual control condition), and these blocks were interspersed with fixation-cross control blocks. Each block consisted of 5 trials of 1 face stimulus type (A, F, N) presented for $4 \mathrm{~s}$ each. Each stimulus was presented once. The face stimuli were counterbalanced for gender (male and female). Each set of face stimuli was randomly ordered within their respective blocks, and blocks were randomly ordered within each functional run as well as across the two functional runs. During the presentation of each face stimulus, subjects identified the valence (positive, negative, neutral) expressed on each face via right-hand button press. 


\section{Functional MRI Data Acquisition}

The fMRI data were acquired using a Philips Achieva Quasar 3T MRI scanner at the Brain Research Imaging Center at the University of Chicago. A structural MRI was obtained with a T1-weighted gradient-echo sequence (301 sagittal slices, repetition time $/ \mathrm{TR}=7.1 \mathrm{~ms}$, echo time $/ \mathrm{TE}=3.4 \mathrm{~ms}$; flip angle $/ \mathrm{FA}=8^{\circ}$, field of view $/ \mathrm{FOV}=250 \times 250 \mathrm{~mm}$, slice thickness $=0.6 \mathrm{~mm}$ ). The fMRI images were obtained with high-field functional MRI utilizing T2*-weighted gradientecho echo planar imaging (EPI) sensitive to the BOLD (blood oxygenation level dependent) signal $(\mathrm{TE}=25 \mathrm{~ms}, \mathrm{TR}=2000$ $\mathrm{ms}, \mathrm{FA}=80^{\circ}, \mathrm{FOV}=230 \times 230 \mathrm{~mm}, 304 \mathrm{~mm}$ axial slices approximately parallel to the AC-PC line, $0.5 \mathrm{~mm}$ slice gap).

\section{Statistical Analysis}

Behavioral and self-report data. Accuracy (percentage) and Reaction Time (ms) data were analyzed in a repeatedmeasure ANOVA, with group as between-subject and Drug (escitalopram/placebo) and Emotion (Anger/Fear/Neutral) as within-subject variables. Subjective mood ratings were analyzed separately per emotion (happy, angry, sad, fear, calm, and irritable) with the within-subject factors Time (before and after administration, $+105 \mathrm{~min}$ ) and Drug and between-subject factor Group.

\section{Functional MRI}

Preprocessing. The preprocessing started with skull stripping of the structural images using BET (Smith, 2002) and subsequent preprocessing was carried out in SPM8 (Wellcome Department of Cognitive Neurology, London; www.fil. ion.ucl.ac.uk/spm); the EPIs were realigned to correct for head motion, the structural image co-registered to the mean EPI image and segmented and normalized to MNI space, EPI images were normalized to the MNI space, and a Gaussian spatial smoothing kernel of $6 \mathrm{~mm}$ FWHM was applied.

Statistics. The subject-level statistical analyses were performed within the general linear model framework (Friston et al, 1994) implemented in SPM8. The preprocessed images were filtered using a 128-s high-pass filter. The regression model consisted of each stimulus type convolved with the canonical hemodynamic response function as well as six motion parameters to correct for residual motion effects. Statistical parametric maps (SPMs) were produced from linear contrasts of interest: each face category versus baseline (eg, angry $>$ fixation) and all faces $>$ fixation. We opted for this latter contrast to use in the group-level analyses for several reasons: (1) because of the application of high-pass filtering, condition (face expression)-specific effects are partly removed; (2) amygdala activation to the 'neutral' expressionless faces have been shown to also activate the amygdala to the same extent as emotional faces (Fitzgerald et al, 2006); and (3) IED subjects have been shown to mislabel 'neutral' faces as conveying negative emotions (Best et al, 2002).

To test whole-brain voxel-wise effects, a full-factorial model was set up in SPM. We investigated the overall effect of face processing (regardless of group or drug, using a voxelwise FWE rate of $P<0.05$ ), main effect of Group, and main effect of Drug and Group $\times$ Drug interaction. The false positive rate for these tests was controlled by applying an initial cluster threshold of $P<0.001$, cluster size $>10$ voxels, and a cluster-level family-wise error (FWE) rate of $P<0.05$. Although the focus is on the 'all faces $>$ baseline' contrast, in order to consider possible emotion-specific effects, the contrast estimates for each emotion separately were also extracted and included in a new model with Emotion as an additional within-subject factor, and reported when showing a significant interaction effect with Group and Drug. For the hypothesis regarding an interaction effect in the amygdala, an initial uncorrected $P<0.005$, cluster size $>5$ voxels was applied, as well as a small-volume, cluster-level FWE rate of $P<0.05$ (amygdala was defined using the Harvard-Oxford subcortical Atlas $70 \%$ probability for the amygdala, implemented in FSL; Smith et al, 2004).

Brain-behavioral correlations. Correlational analyses were performed to test whether parameter estimates of significant clusters for faces $>$ baseline contrast $($ Group $\times$ Drug interaction) were linearly related to behavioral measures of aggression (LHA), psychopathy (PCL), and impulsivity (BIS-11 and LHIB).

Decoding analysis. In order to aid the interpretation of the whole-brain voxel-wise analysis, we applied a novel method to 'decode' significant clusters using Neurosynth (Yarkoni et al, 2011). This method essentially correlates an input image with reverse inference maps related to a topic (ie, a map of the probability per voxel of being active given a certain topic, $\mathrm{cf}$. Chang et al, 2012). The analysis provides an automatic 'interpretation of the involvement' of an input image (for instance, a specific brain region or a contrast image of different conditions) in a certain a topic (eg, 'emotion'). The analysis was restricted to a set of 140 latent topics as presented in Poldrack et al (2012).

\section{RESULTS}

\section{Self-Report Mood Ratings}

The self-report showed that controls overall rated themselves as higher on happiness $(\mathrm{F}(1,28)=13.8, P=0.001)$ and calmness $(\mathrm{F}(1,28)=7.4, P=0.011)$ compared to IED, but not on any of the other emotions (all $P>0.15$ ). No significant Group $\times$ Drug $\times$ Time interaction effects were found (all $P>0.08)$.

\section{Behavioral Results}

Accuracy during the emotion detection was high overall $(>78 \%)$, and a significant main effect of Emotion was observed $(\mathrm{F}(2,58)=4.45, P=0.016$; neutral (mean $(\mathrm{SE}), 78 \%$ (3.6\%); fear, $87 \%(3.1 \%)$; anger, $84 \%(2.9 \%)$; both fear $>$ neutral and fear $>$ anger, $P<0.05)$ but no other main or interaction effects were found (all $P>0.05$ ). Reaction time data showed a similar pattern: a significant main effect of Emotion $(\mathrm{F}(2,56)=9.53, \quad P=0.001)$. Reaction times for neutral faces were higher than anger or fear (neutral, 1233 (33.0); fear, 1100.0 (43.4); anger, 1134.91 (39.3); both fear $>$ neutral and anger $>$ neutral, $P<0.01)$. No other significant main or interaction effects were found. 
Table 2 Whole-Brain Results for the Faces > Fixation Contrast

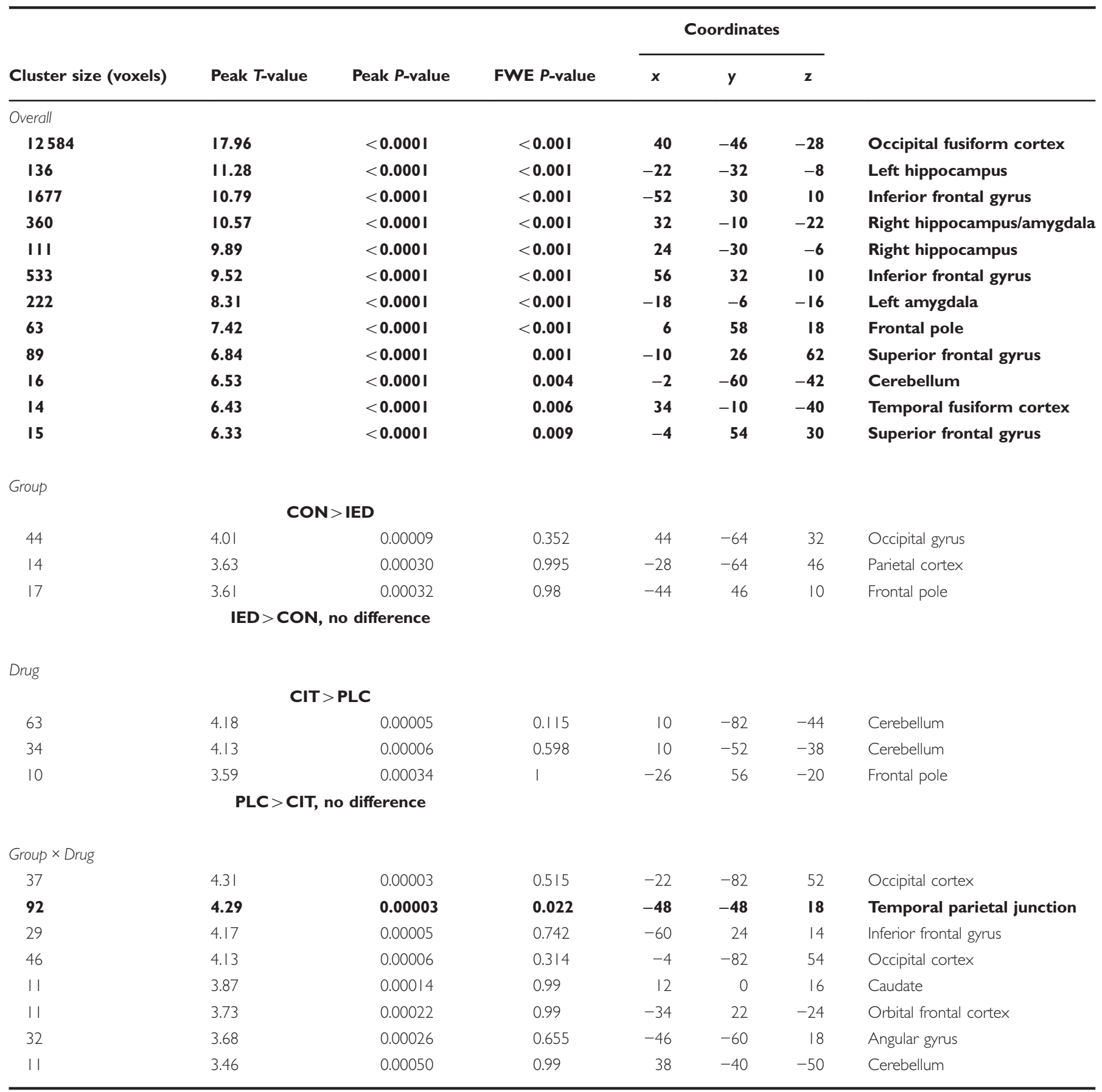

Overall effect across the two groups and drugs conditions are FWE voxel-wise corrected $P<0.05$. For the main effect of Group (Controls $>$ IED and IED $>$ controls), Drug (CIT $>$ PLC, and PLC $>C I T)$, and Group $\times$ Drug Interaction, clusters that met a $P<0.001$ uncorrected, cluster size $>10$ voxels criterion are presented for descriptive purposes, and clusters are printed in bold that met a whole-brain FWE cluster corrected $P<0.05$ threshold.

\section{fMRI Results}

Whole-brain analyses on the faces $>$ baseline contrast showed a significant main effect in several well-known face processing regions: visual cortex, bilateral amygdala/hippocampus, superior temporal gyrus, and inferior frontal gyrus (see Table 2). No significant cluster-corrected effects of the factors Group or Drug were found (all $P<0.001$ uncorrected, cluster size $>10$ voxels, results are reported in Table 2). The Group $\times$ Drug interaction test revealed an effect in the left temporal parietal junction (TPJ; see Figure 1). The decoding (Neurosynth) analysis showed that the two latent topics that are most strongly linked to the TPJ were related to 'social cognition' and 'moral beliefs' (see Supplementary Table S2 for the complete list of results). The region of interest analysis of the amygdala revealed a Group $\times$ Drug interaction 

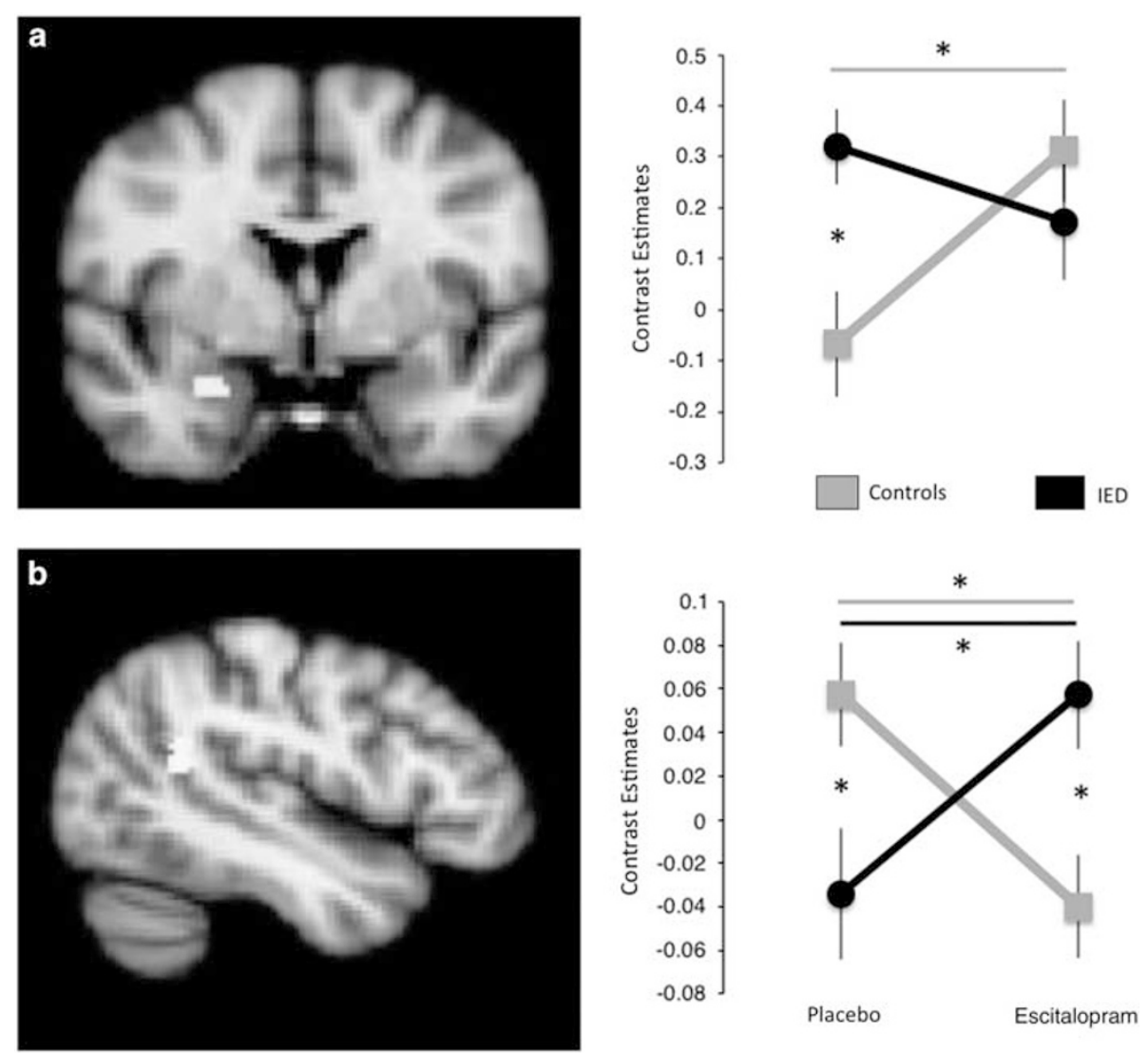

Figure I Group $\times$ Drug interaction effects. (a) Right amygdala, small volume corrected. (b) Left temporal parietal junction. Error bars are SEM (radiological convention; left is right, right is left). *Post hoc pairwise t-test $P<0.05$ (between-group effect per drug condition, or within-group change because of drug condition).

effect, small-volume, cluster-level FWE $P<0.05$ ( $x, y$, $z=30 / 0 /-22$, cluster size $=20$ voxels, $\left.P_{\text {unc }}=0.001\right)$. No significant correlations were observed between the amygdala or TPJ (contrast estimates for citalopram $>$ placebo and placebo only) and behavioral measures of aggression, psychopathy, and impulsivity.

\section{DISCUSSION}

In this study, we investigated how a selective serotonin reuptake inhibitor, escitalopram, affects the neural mechanisms of social-emotional processing in IED. We found that escitalopram increased amygdala activity during socialemotional processing in controls, but surprisingly not in IED. In addition to the amygdala results, we also found a strong and intriguing effect in the TPJ: the IED group displayed increased activity on escitalopram (relative to placebo) compared with healthy controls. This finding is of great interest, as the TPJ is associated with social-cognitive processes, such as perspective taking and empathy (Van Overwalle and Baetens, 2009), and the decoding analysis confirmed the relatively specific involvement of the TPJ in such social-cognitive processes. Therefore, in addition to dysfunctional amygdala-prefrontal circuitry (Coccaro et al, 2007), our findings suggest that the TPJ is also a potentially important brain region involved in IED and escitalopram may reduce aggressive tendencies toward others in IED by enhancing social-cognitive processing, rather than affecting the primary salience response of the amygdala.

The amygdala is perhaps the most well-established region in face processing (Morris et al, 1998; Vuilleumier et al, 2001; Fusar-Poli et al, 2009) and a main target of dorsal raphe nucleus serotonergic projections (Maier et al, 2006; Asan et al, 2013). Previously, IED was found to be associated with a dysbalance in amygdala and OFC activity during threatening face processing (Coccaro et al, 2007). Contrary to our expectations, here we did not find evidence that citalopram decreases amygdala activation in IED. Moreover, although the results in controls (escitalopram increases amygdala activation) are in line with a prior report (Bigos et al, 2008), some studies did not observe an effect of citalopram on amygdala activity (Brühl et al, 2010; Henry et al, 2013) and several other single-dose and short-term citalopram administration studies found amygdala decreases (Del-Ben et al, 2005; Harmer et al, 2006; Anderson et al, 2007; Arce et al, 2008; Murphy et al, 2009; Windischberger et al, 2010). Although it is possible that our results in controls are perhaps due to sampling error (see Limitations section), several other methodological issues in citalopram administration studies also need to be considered. The citalopram fMRI studies differ substantially on potentially interacting factors like administration method (oral or intravenous), design (within or between subjects), duration of the drug administration (eg, a single dose or a daily intake for several days), dose, task (eg, block design or event related, masked 
stimuli or unmasked stimuli), and task contrast (eg, comparing faces with a low-level baseline or emotionspecific effects such as fear $>$ happy). Hence, any of these factors could turn out to be a major determinant on itself or interact with other factors in the effect that citalopram has on amygdala activity and social-emotion processing; a topic that deserves systematic investigation. Specifically, given the delay in SSRI effectiveness (Harmer et al, 2009), the difference between single-dose, short-term (eg, 1 week), and longlasting effects (months) of SSRIs on brain systems seems crucial and, ideally, future research should also address longterm effects of SSRI on amygdala functioning in IED.

Serotonin is widely distributed in the brain (Cools et al, 2008) and linked to a variety of cognitive functions related to aversive processing (Cools et al, 2008; Dayan and Huys, 2009). In addition, serotonin dysfunction affects social cognition and behavior; for instance, animal research has shown that serotonin suppresses reactive aggression and promotes affiliate actions linked to social status in primates (Dayan and Huys, 2009). Moreover, human research has shown that tryptophan depletion (that decreases 5-HT availability) reduces cooperative behavior in a prisoner's dilemma game (Wood et al, 2006), whereas citalopram administration made subjects more likely to judge harmful actions as forbidden (Crockett et al, 2010) and enhanced reward sensitivity to facial expressions of a social partner (Tse et al, 2014). FMRI studies have found that citalopram reduces negative self-referential processing in depressed patients by enhancing medial PFC activity (Di Simplicio et al, 2012), and normalizes resting-state connectivity with the dorsal medial PFC (McCabe et al, 2011). Given the variety of lines of evidence on the link between socialcognition and serotonin, it is worth considering this a potential therapeutic mechanism, perhaps even more so than through amygdala-based emotional salience processing. It is important to point out that beyond serotonin, (impulsive) aggression is related to a number of neurotransmitter and neuropeptide systems, including dopamine, GABA, norepinephrine, vasopressin, and oxytocin (Yanowitch and Coccaro, 2011) that can be additional useful targets for pharmacological studies and treatment.

The meta-analytic decoding results of the TPJ revealed a relatively specific involvement with social-cognition. This notion is further underscored by a transcranial direct current stimulation study showing that TPJ stimulation increased perspective taking (Santiesteban et al, 2012). The link between TPJ functioning and social-cognitive processes in IED moreover fits with deficiencies in the neural mechanisms of social-cognitive functioning during perspective taking, empathy, and theory-of-mind tasks in psychopathy (Anderson and Kiehl, 2012; Blair and Lee, 2013; Decety et al, 2013a) that is also clearly characterized by aggressive behavior, although to a larger extend premeditated rather than impulsive. It is of note however that the current sample scored substantially lower on the PCL than the cutoff for psychopathology (Decety et al, 2013b) and more research is needed to further investigate the differences and similarities in the neural mechanism of social-cognitive functioning between IED and psychopathy.

Citalopram is an often-prescribed drug for not only depression (Cipriani et al, 2009) but also various other disorders like PTSD (Hetrick et al, 2010) and OCD (Soomro et al, 2008). To understand the potential efficiency of a drug, it is important to uncover its neural mechanism of action. Previous work has shown reasonable effectiveness of another SSRI, fluoxetine, in treating IED (Coccaro et al, 2009), and hence the current findings indicate a potential neural mechanism for SSRI functioning. The results suggest that escitalopram may affect the neural mechanisms of social-emotional and social-cognitive processes. Our findings aid in the understanding of the mechanisms of treatment efficiency obtained from multicomponent cognitive behavioral therapy (McCloskey et al, 2008). However, the effectiveness of escitalopram for IED treatment, and a detailed mechanistic model, will ultimately have to be tested in large randomized controlled trials.

\section{LIMITATIONS}

In addition to the above-mentioned methodological consideration with respect to the amygdala findings, there are some other limitations of this study and the findings that need to be taken into account. For instance, it has been found that the test-retest reliability of the influence of citalopram administration on amygdala activity is low (Klomp et al, 2013), perhaps because of the relatively low test-retest reliability of amygdala activity to emotional faces in general (Plichta et al, 2012). Another critical consideration, as with many studies in the field of clinical fMRI, is the small sample size of this study that negatively affects the reliability of the findings (Button et al, 2013; Yarkoni, 2009).

\section{CONCLUSIONS}

This study's findings suggest that escitalopram may enhance social-emotional functioning in IED, and hence provide insight into potential therapeutic mechanisms. Future research should further elucidate the long-term effects of SSRIs on various social-cognitive tasks in IED.

\section{FUNDING AND DISCLOSURE}

Dr Coccaro's work has been funded by the NIMH grant R21 MH083198. Dr Coccaro reports that he is on the Scientific Advisory Board of Azevan Pharmaceuticals and that he has stock options in Azivan Pharmaceuticals through this role. Dr Lee reports that he has received a research grant from Azivan Pharmaceuticals. The remaining authors declare no conflict of interest.

\section{REFERENCES}

Anderson IM, Del-Ben CM, McKie S, Richardson P, Williams SR, Elliott $\mathrm{R}$ et al (2007). Citalopram modulation of neuronal responses to aversive face emotions: a functional MRI study. Neuroreport 18: 1351-1355.

Anderson NE, Kiehl KA (2012). The psychopath magnetized: insights from brain imaging. Trends Cogn Sci 16: 52-60.

Arce E, Simmons AN, Lovero KL, Stein MB, Paulus MP (2008). Escitalopram effects on insula and amygdala BOLD activation during emotional processing. Psychopharmacology 196: 661-672.

Asan E, Steinke M, Lesch K-P (2013). Serotonergic innervation of the amygdala: targets, receptors, and implications for stress and anxiety. Histochem Cell Biol 139: 785-813. 
Barratt ES (1965). Factor analysis of some psychometric measures of impulsiveness and anxiety. Psychol Rep 16: 547-554.

Best M, Williams JM, Coccaro EF (2002). Evidence for a dysfunctional prefrontal circuit in patients with an impulsive aggressive disorder. Proc Natl Acad Sci USA 99: 8448-8453.

Bigos KL, Pollock BG, Aizenstein HJ, Fisher PM, Bies RR, Hariri AR (2008). Acute 5-HT reuptake blockade potentiates human amygdala reactivity. Neuropsychopharmacology 33: 3221-3225.

Blair RJR, Lee TMC (2013). The social cognitive neuroscience of aggression, violence, and psychopathy. Soc Neurosci 8: 108-111.

Brühl AB, Kaffenberger T, Herwig U (2010). Serotonergic and noradrenergic modulation of emotion processing by single dose antidepressants. Neuropsychopharmacology 35: 521-533.

Button KS, Ioannidis JPA, Mokrysz C, Nosek BA, Flint J, Robinson ESJ et al (2013). Power failure: why small sample size undermines the reliability of neuroscience. Nat Rev Neurosci 14: 365-376.

Chang LJ, Yarkoni T, Khaw MW, Sanfey AG (2012). Decoding the role of the insula in human cognition: functional parcellation and large-scale reverse inference. Cereb Cortex 23: 739-749.

Cipriani A, Santilli C, Furukawa TA, Signoretti A, Nakagawa A, McGuire $\mathrm{H}$ et al (2009). Escitalopram versus other antidepressive agents for depression. Cochrane Database Syst Rev (2): CD006532.

Coccaro EF (1989). Central serotonin and impulsive aggression. $\mathrm{Br} \mathrm{J}$ Psychiatry 8: 52-62.

Coccaro EF (2000). Intermittent explosive disorder. Curr Psychiatry Rep 2: 67-71.

Coccaro EF (2012). Intermittent explosive disorder as a disorder of impulsive aggression for DSM-5. Am J Psychiatry 169: 577-588.

Coccaro EF, Berman ME, Kavoussi RJ (1997). Assessment of life history of aggression: development and psychometric characteristics. Psychiatry Res 73: 147-157.

Coccaro EF, Lee R, Kavoussi RJ (2010). Aggression, suicidality, and intermittent explosive disorder: serotonergic correlates in personality disorder and healthy control subjects. Neuropsychopharmacology 35: 435-444.

Coccaro EF, Lee R, McCloskey MS (2014). Validity of the new A1 and A2 criteria for DSM-5 intermittent explosive disorder. Comprehensive Psychiatry 55: 260-267.

Coccaro EF, Lee RJ, Kavoussi RJ (2009). A double-blind, randomized, placebo-controlled trial of fluoxetine in patients with intermittent explosive disorder. J Clin Psychiatry 70: 653-662.

Coccaro EF, McCloskey MS, Fitzgerald DA, Phan KL (2007). Amygdala and orbitofrontal reactivity to social threat in individuals with impulsive aggression. Biol Psychiatry 62: 168-178.

Coccaro EF, Schmidt-Kaplan CA (2012). Life history of impulsive behavior: development and validation of a new questionnaire. J Psychiatr Res 46: 346-352.

Cools R, Roberts AC, Robbins TW (2008). Serotoninergic regulation of emotional and behavioural control processes. Trends Cogn Sci 12: $31-40$.

Crockett MJ, Clark L, Hauser MD, Robbins TW (2010). Serotonin selectively influences moral judgment and behavior through effects on harm aversion. Proc Natl Acad Sci USA 107: 17433-17438.

Dayan P, Huys QJM (2009). Serotonin in affective control. Annu Rev Neurosci 32: 95-126.

Decety J, Chen C, Harenski C, Kiehl KA (2013a). An fMRI study of affective perspective taking in individuals with psychopathy: imagining another in pain does not evoke empathy. Front Hum Neurosci 7: 489.

Decety J, Skelly LR, Kiehl KA (2013b). Brain response to empathyeliciting scenarios involving pain in incarcerated individuals with psychopathy. JAMA Psychiatry 70: 638-645.

Del-Ben CM, Deakin JFW, McKie S, Delvai NA, Williams SR, Elliott $\mathrm{R}$ et al (2005). The effect of citalopram pretreatment on neuronal responses to neuropsychological tasks in normal volunteers: an FMRI study. Neuropsychopharmacology 30: 1724-1734.
Di Simplicio M, Norbury R, Harmer CJ (2012). Short-term antidepressant administration reduces negative self-referential processing in the medial prefrontal cortex in subjects at risk for depression. Mol Psychiatry 17: 503-510.

Ekman P, Friesen WV, (1976). Consulting Psychologists Press: Pictures of Facial Affect Palo Alto.

First MB, Spitzer RL, Gibbon M, Williams J (1997). Structured Clinical Interview for DSM-IV Axis I Disorders (SCID-I), Clinician Version, Administration Booklet. Biometrics Research, New York State Psychiatric Institute: New York.

Fitzgerald DA, Angstadt M, Jelsone LM, Nathan PJ, Phan KL (2006). Beyond threat: amygdala reactivity across multiple expressions of facial affect. Neuroimage 30: 1441-1448.

Frankle WG, Lombardo I, New AS, Goodman M, Talbot PS, Huang $Y$ et al (2005). Brain serotonin transporter distribution in subjects with impulsive aggressivity: a positron emission study with [11C] McN 5652. Am J Psychiatry 162: 915-923.

Friston KJ, Holmes AP, Worsley KJ, Poline JP, Frith CD, Frackowiak RSJ (1994). Statistical parametric maps in functional imaging: a general linear approach. Hum Brain Mapp 2: 189-210.

Fusar-Poli P, Placentino A, Carletti F, Landi P, Allen P, Surguladze $S$ et al (2009). Functional atlas of emotional faces processing: a voxel-based meta-analysis of 105 functional magnetic resonance imaging studies. J Psychiatry Neurosci 34: 418-432.

George DT, Phillips MJ, Lifshitz M, Lionetti TA, Spero DE, Ghassemzedeh N et al (2011). Fluoxetine treatment of alcoholic perpetrators of domestic violence: a 12-week, double-blind, randomized, placebo-controlled intervention study. J Clin Psychiatry 72: 60-65.

Gur RC, Sara R, Hagendoorn M, Marom O, Hughett P, Macy L et al (2002). A method for obtaining 3-dimensional facial expressions and its standardization for use in neurocognitive studies. J Neurosci Methods 115: 137-143.

Harmer CJ, Goodwin GM, Cowen PJ (2009). Why do antidepressants take so long to work? A cognitive neuropsychological model of antidepressant drug action. $\mathrm{Br} J$ Psychiatry 195: 102-108.

Harmer CJ, Mackay CE, Reid CB, Cowen PJ, Goodwin GM (2006). Antidepressant drug treatment modifies the neural processing of nonconscious threat cues. Biol Psychiatry 59: 816-820.

Hart SD, Cox DN, Hare RD (1995). The Hare Psychopathy Checklist: Screening Version. Multi-Health Systems: Toronto.

Henry ME, Lauriat TL, Lowen SB, Churchill JH, Hodgkinson CA, Goldman D et al (2013). Effects of citalopram and escitalopram on fMRI response to affective stimuli in healthy volunteers selected by serotonin transporter genotype. Psychiatry Res 213: 217-224.

Hetrick SE, Purcell R, Garner B, Parslow R (2010). Combined pharmacotherapy and psychological therapies for post traumatic stress disorder (PTSD). Cochrane Database Syst Rev (7): CD007316.

Klomp A, van Wingen GA, de Ruiter MB, Caan MWA, Denys D, Reneman L (2013). Test-retest reliability of task-related pharmacological MRI with a single-dose oral citalopram challenge. NeuroImage 75: 108-116.

Lee RJ, Gill A, Chen B, McCloskey M, Coccaro EF (2012). Modulation of central serotonin affects emotional information processing in impulsive aggressive personality disorder. J Clin Psychopharmacol 32: 329-335.

Lesch K-P, Merschdorf U (2000). Impulsivity, aggression, and serotonin: a molecular psychobiological perspective. Behav Sci Law 18: 581-604.

Maier SF, Amat J, Baratta MV, Paul E, Watkins LR (2006). Behavioral control, the medial prefrontal cortex, and resilience. Dialogues Clin Neurosci 8: 397-406.

McCabe C, Mishor Z, Filippini N, Cowen PJ, Taylor MJ, Harmer CJ (2011). SSRI administration reduces resting state functional connectivity in dorso-medial prefrontal cortex. Mol Psychiatry 16: 592-594. 
McCloskey MS, Noblett KL, Deffenbacher JL, Gollan JK, Coccaro EF (2008). Cognitive-behavioral therapy for intermittent explosive disorder: a pilot randomized clinical trial. J Consult Clin Psychol 76: 876-886.

Morris JS, Friston KJ, Büchel C, Frith CD, Young AW, Calder AJ et al (1998). A neuromodulatory role for the human amygdala in processing emotional facial expressions. Brain 121: 47-57.

Murphy SE, Norbury R, O'Sullivan U, Cowen PJ, Harmer CJ (2009). Effect of a single dose of citalopram on amygdala response to emotional faces. Br J Psychiatry 194: 535-540.

Pfohl B, Blum N, Zimmerman M (1997). Structured Interview for DSM-IV Personality. University of Iowa College of Medicine: Iowa.

Plichta MM, Schwarz AJ, Grimm O, Morgen K, Mier D, Haddad L et al (2012). Test-retest reliability of evoked BOLD signals from a cognitive-emotive fMRI test battery. NeuroImage 60: 1746-1758.

Poldrack RA, Mumford JA, Schonberg T, Kalar D, Barman B, Yarkoni $\mathrm{T}$ (2012). Discovering relations between mind, brain, and mental disorders using topic mapping. PLoS Comput Biol 8: e1002707.

Santiesteban I, Banissy MJ, Catmur C, Bird G (2012). Enhancing social ability by stimulating right temporoparietal junction. Curr Biol 22: 2274-2277.

Silva H, Iturra P, Solari A, Villarroel J, Jerez S, Jiménez $M$ et al (2010). Fluoxetine response in impulsive-aggressive behavior and serotonin transporter polymorphism in personality disorder. Psychiatr Genet 20: 25-30.

Smith SM (2002). Fast robust automated brain extraction. Hum Brain Mapp 17: 143-155.

Smith SM, Jenkinson M, Woolrich MW, Beckmann CF, Behrens TEJ, Johansen-Berg $\mathrm{H}$ et al (2004). Advances in functional and structural MR image analysis and implementation as FSL. Neuroimage 23: S208-S219.
Soomro GM, Altman D, , Rajagopal S, Oakley-Browne M (2008). Selective serotonin re-uptake inhibitors (SSRIs) versus placebo for obsessive compulsive disorder (OCD). Cochrane Database Syst Rev (1): CD001765.

Tse WS, Chow H, Wing YK, Bond AJ (2014). Using a partner's facial emotion to elucidate social dominance motivation induced by an SSRI. Eur Neuropsychopharmacol 24: $1641-1649$

Van Overwalle F, Baetens K (2009). Understanding others' actions and goals by mirror and mentalizing systems: a meta-analysis. Neuroimage 48: 564-584.

Vuilleumier P, Armony JL, Driver J, Dolan RJ (2001). Effects of attention and emotion on face processing in the human brain: an event-related fMRI study. Neuron 30: 829-841.

Windischberger C, Lanzenberger R, Holik A, Spindelegger C, Stein P, Moser U et al (2010). Area-specific modulation of neural activation comparing escitalopram and citalopram revealed by pharmaco-fMRI: a randomized cross-over study. Neuroimage 49: 1161-1170.

Wood RM, Rilling JK, Sanfey AG, Bhagwagar Z, Rogers RD (2006). Effects of tryptophan depletion on the performance of an iterated prisoner's dilemma game in healthy adults. Neuropsychopharmacology 31: 1075-1084.

Yanowitch R, Coccaro EF (2011). The neurochemistry of human aggression. Adv Genet 75: 151-169.

Yarkoni T (2009). Big correlations in little studies: inflated fMRI correlations reflect low statistical power-commentary on Vul et al. (2009). Persp Psychol Sci 4: 294-298.

Yarkoni T, Poldrack RA, Nichols TE, Van Essen DC, Wager TD (2011). Large-scale automated synthesis of human functional neuroimaging data. Nat Methods 8: 665-670.

Supplementary Information accompanies the paper on the Neuropsychopharmacology website (http://www.nature.com/npp) 